02

\title{
Molecular Structure, Vibrational Spectra, Molecular Docking and ADMET Study of Cellulose Triacetate II*
}

\author{
(C) Sefa Celik ${ }^{1}$, A. Demet Demirag ${ }^{2}$, Aysen E. Ozel ${ }^{1}$, and Sevim Akyuz ${ }^{3}$ \\ ${ }^{1}$ Istanbul University, Faculty of Science, Department of Physics, Vezneciler, \\ 34134 Istanbul, Turkey \\ ${ }^{2}$ Internet Network and Technologies Department, Vocational School, Yeditepe university, \\ Istanbul, Turkey \\ ${ }^{3}$ Istanbul Kultur University, Faculty of Science and Letters, Department of Physics, \\ 34156 Bakirkoy-Istanbul, Turkey \\ e-mail: scelik@istanbul.edu.tr
}

Received May 15, 2019

Revised March 19, 2020

Accepted March 28, 2020

People have started to look for alternative sources because of the health problems created by petrochemical products used in all areas of human life and environmental problems that remain intact in nature for years. In this study, molecular structure analysis of cellulose triacetate II (CTA II) molecule, obtained from cellulose II and acetate, was carried out. There is an important relationship between the structure and activity of molecules, so it is very important to determine the geometric structure of a molecule. Therefore, using density functional theory (DFT) the most stable molecular geometries of the cellulose triacetate II monomer $\left(\mathrm{C}_{12} \mathrm{H}_{18} \mathrm{O}_{9}\right)$ as well as dimer $\left(\mathrm{C}_{24} \mathrm{H}_{36} \mathrm{O}_{18}\right)$, which included intermolecular H-bonding, were calculated. The analogous calculations were carried out for the (CTA-II $)_{2}$ nano-cluster $\left(\mathrm{C}_{24} \mathrm{H}_{34} \mathrm{O}_{17}\right)$, which represents the local structure of CTA-II crystal, and created by binding the two most stable CTA II molecules by covalent bond. Scaled wavenumbers and potential energy distribution of the vibrational modes of CTA monomer and (CTA-II $)_{2}$ nano-cluster were computed. In order to evaluate the interaction of CTA II with the Aspergillus niger cellulase enzyme, which is an important that is active in cellulose digestion and CTA II, molecular docking studies were carried out. H-binding interactions between CTA II (in monomeric, dimeric and cluster forms) and the active site of the Aspergillus niger cellulase enzyme were shown. Moreover, in silico ADMET prediction study was calculated for CTA-II monomer to predict its druglikeness properties.

Keywords: Cellulose triacetate II, Density Function Theory, IR-ATR, cluster for.

* Полный текст статьи опубликован в „Optics and Spectroscopy“ 2020 V. 128. N 8. 